Рогулин Родион Сергеевич, Максименко Валерий Иванович, Жандармов Владимир Олегович, Пугачева Ева Сергеевна, Матвеев Владислав Викторович, Рогулин Данила Сергеевич

\title{
О РАСШИРЕНИИ СКЛАДСКОЙ БАЗЫ В РАССМАТРИВАЕМОМ ЭКОНОМИЧЕСКОМ РАЙОНЕ С ПАРАЛЛЕЛЬНЫМ ОПРЕДЕЛЕНИЕМ ОПТИМАЛЬНОЙ РАБОТЫ ЛОГИСТИКИ
}

В статье предложено комплексное решение трех задач линейного программирования: транспортиая задача (проблема логистики), задача о максимальном потоке, задача размецения чентров. Подобные задачи в предложенной комплексной постановке часто возникают на предприятиях в проиессе принятия решения о расширении складской базы с последуюиим определением оптимального процесса работы складов как пунктов отправки груза до потребителя. Рассмотрены основные алгоритмы поиска оптимального речения, сформулирована комплексная задача, построена модель и реализован алгоритм решения, проведено сравнение существуощего метода и авторского. Предложенная модель может быть использована на любом предприятии, где необходимо найти оптимальный комбинаторньй вариант для доставки со склада с иелью минимизации затрат на транспортировку готовой продукции. Такая задача в точности подходит к экономической ситуации, когда предприятию предстоит отправить продукцию по известной транспортной инфраструктуре при учете стоимости на перевозку. Задача, которую мы решаем, впервые появилась на предприятии лесоперерабатывающей направленности. Такая проблема носит характер нетривиально комбинаторньй.

Ключевые слова: математическое моделирование, линейное программирование, максимальный поток, разиещение центров, производство, логистика.

\section{Rodion Rogulin, Valery Maksimenko, Vladimir Zhandarmov, Eva Pugacheva, Vladislav Matveyev, Danila Rogulin ON THE EXPANSION OF THE WAREHOUSE BASE \\ IN THE CONSIDERED ECONOMIC AREA WITH PARALLEL DETERMINATION OF THE OPTIMAL LOGISTICS WORK}

This article proposes a comprehensive solution of three linear programming problems: Transport problem (logistics problem), Maximum flow problem, Center allocation problem. Similar tasks in the proposed complex formulation often arise at enterprises in the process of making decisions on expanding the warehouse base with the subsequent determination of the optimal process for the operation of warehouses as points of shipment to the consumer The basic algorithms for finding optimal solutions are considered, a complex task is formulated, a model is built and a solution algorithm is implemented, and comparison of the existing method and author's method is carried out. The proposed model can be used in any enterprise where it is necessary to find the best combinatory variant for delivery in order to minimize the cost of transporting of the finished product. Such a task corresponds exactly to the economic situations when an enterprise will have to deliver products using a known transport infrastructure, taking into account the cost of transportation. The task that we have solved appeared for the first time at a timber processing enterprise. Such a problem is non-trivial combinatorial.

Key words: mathematical modeling, linear programming, maximum flow, center allocation, production, logistics.

Bведениe / Introduction. Каждое предприятие в ходе хозяйственной деятельности ставит главной задачей минимизацию издержек в процессе транспортировки. При всем многообразии методов оптимизации процессов управления ресурсами предприятий в научной литературе недостаточно представлены единые алгоритмы и модели для нахождения оптимального решения комплексных проблем хозяйственной деятельности предприятия. На любом предприятии существуют 
следующие основные задачи предприятий: задача производства (оптимальный выпуск продукции), транспортная задача (определение пути и объема перевозок по двудольным графам), задача максимального потока (нахождение максимального по объему перевозок пути на графе), задача минимизации времени, задача о размещении центров сбыта (обслуживания), задача распределения людских ресурсов при производстве. В нашей статье мы рассмотрим только три из них: транспортную задачу, задачу размещения центров, задачу о максимальном потоке - как единую комплексную задачу.

При имеющихся денежных транспортных издержках необходимо найти оптимальный объем перевозки продукции напрямую. Такая задача получила распространение в литературе как транспортная задача [1].

Существует помимо этой задачи и другая, не менее сложная, с точки зрения трудозатрат на ее решения: задача размещения центров [2] - в этой задаче ставится вопрос об определении расположения складов в рассматриваемом районе так, чтобы расстояние от всех грузополучателей до складов было минимально. Ограничения описаны в [4].

Стоит отметить еще одну проблему - задача о максимальном потоке. Цель - найти максимально возможный поток на графе с учетом пропускном способности графа и объема, перевозимого по графу. Рассмотрим ряд методов и моделей, которые решают данные задачи (таблица 1).

Таблича 1

Методы и модели решения поставленной задачи

\begin{tabular}{|c|c|}
\hline Факторы & Описание стратегии \\
\hline $\begin{array}{l}\text { Квадратическое } \\
\text { программирование } \\
(\text { КП) }[1,2]\end{array}$ & $\begin{array}{l}\text { Составляется отдельная квадратическая модель, после работы стандартных ал- } \\
\text { горитмов КП, представляется ответ к задаче в виде одномерного массива. }\end{array}$ \\
\hline $\begin{array}{l}\text { Supply Chain } \\
\text { Management (SCM) [2] }\end{array}$ & $\begin{array}{l}\text { Управленческая концепция и организационная стратегия, заключающаяся в ин- } \\
\text { тегрированном подходе к планированию и управлению всем потоком информа- } \\
\text { ции о сырье, материалах, продуктах, услугах, возникающих и преобразующихся } \\
\text { в логистических и производственных процессах предприятия, нацеленном на } \\
\text { измеримый совокупный экономический эффект (снижение издержек, удовлет- } \\
\text { ворение спроса на конечную продукцию). Концепция основана на генетическом } \\
\text { алгоритме. }\end{array}$ \\
\hline $\begin{array}{l}\text { Генетический } \\
\text { алгоритм [1] }\end{array}$ & $\begin{array}{l}\text { Эвристический алгоритм поиска, который используется для поиска решения } \\
\text { задач оптимизации и моделирования. Стратегия заключается в случайном под- } \\
\text { боре, комбинирования и вариаций изначальных параметров с использованием } \\
\text { механизмов, аналогичных естественному отбору в природе. Является разновид- } \\
\text { ностью эволюционных вычислений, с помощью которых решаются оптимиза- } \\
\text { ционные задачи с использованием методов естественной эволюции, таких как } \\
\text { наследование, мутации, отбор и кроссинговер. Отличительной особенностью } \\
\text { генетического алгоритма является акщент на использование оператора «скре- } \\
\text { щивания», который производит операцию рекомбинации решений-кандидатов, } \\
\text { роль которой аналогична роли скрещивания в живой природе. }\end{array}$ \\
\hline
\end{tabular}

Для решения каждой из вышеперечисленных задач существуют отдельные модели $[1,2]$, но мы предлагаем комплексное решение трех вышеописанных отдельных задач. Под комплексным решением будем понимать единую линейную модель смешано-целочисленного программирования для трех вышеуказанных проблем лесоперерабатывающего комплекса.

Сформулируем обобщенную постановку задачи: определить оптимальный вариант размещения складов так, чтобы стоимость доставки товара покупателю была минимальной. Цель - минимизировать расходы на транспортировку (денежные), количество открытых пунктов складирования [10].

Такая модель может быть полезна для любой логистической компании. 
Данная работа посвящена построению модели, выбору метода и алгоритма поиска решения этой задачи. Все вышеперечисленные задачи сводятся к линейным моделям, что значительно упрощает нахождение оптимального решения, отдельно модели известны в литературе $[1,2]$. Для решения вышеперечисленных задач используются алгоритмы поиска оптимального решения метод отсечения (Гомори) [3].

Maтериаль и методы /Materials and methods. Таюже экзогенно задано время для транспортировки товара из пункта $i$ в $j$, обозначим матрицу временных затрат как

$$
T=\left\{t_{i j}\right\}, i=1: n, j=1: m .
$$

Обозначим матрицу пропускной способности графа как:

$$
D=\left\{d_{i j}\right\}, i=1: n, j=1: m \text {. }
$$

Пусть существует некоторое количество товара, определенное спросом потребителя (магазины, ИП и т. д.). Обозначим его

$$
a=\left\{a_{j}\right\}, j=1: m,
$$

Кроме того, определим затраты на перевозку товара из пункта $i$ в $j$. Обозначим как

$$
C=\left\{c_{i j}\right\}, i=1: n, j=1: m \text {. }
$$

Доопределим входные данные издержками на открытие предприятие в каждом рассмотренном пункте как

$$
f .=\left\{f_{i}\right\}, i=1: n
$$

Максимальное количество пунктов производства определим количеством $Q$. Для полноты набора данных остается определить необходимое количество запасов товаров для доставки потребителю, обозначим их как

$$
b=\left\{b_{i}\right\}, i=1: n .
$$

Транспортная задача. Пусть $x_{i_{1} j_{1}}-$ есть количество товара, перевозимое из пункта $i_{1}$ в пункт $j_{1}[11,12]$.

Необходимо минимизировать расходы, тогда целевая функция примет вид [4]:

$$
\sum_{i_{1}=1}^{n} \sum_{j_{1}=1}^{n} x_{i_{1} j_{1}} c_{i_{1} j_{1}} \rightarrow \min
$$

Учтем спрос потребителя, тогда $[15,16]$ :

$$
\sum_{i_{1}=1}^{n} x_{i_{1} j}=a_{j}, j=1: m
$$

Также учтем запасы на складе, тогда:

$$
\sum_{j_{1}=1}^{n} x_{i j_{1}} \leq b_{i}, i=1: m
$$

Задача о максимальном потоке. Математическая модель подробно представлена в [1].

Задача о размешении центров обслуживания. Математическая модель подробно представлена в [1]. Обозначим задачу $F_{0}$ :

$$
\sum_{j=1}^{m} \sum_{i=1}^{n} c_{i j} x_{i j}+\sum_{i=1}^{n} f_{i} z_{i} \rightarrow \min
$$




$$
\begin{gathered}
k_{i}=\sum_{j=1}^{m} x_{i j}, i=1: n, \\
\sum_{i=1}^{n} k_{i}=\sum a_{j} \\
\sum_{j=1}^{m} x_{i j}=\sum_{i=1}^{n} x_{i j} ; i=1: n, j=1: m, \\
y_{i j} \leq x_{i j}, i=1: n, j=1: m, \\
0 \leq x_{i j} \leq d_{i j}, i=1: n, j=1: m, \\
\sum_{i=1}^{n} x_{i j}=a_{j}, j=1: m, \\
\sum z_{j} \leq Q, \\
z_{i} \in(0 ; 1), i=1: m,
\end{gathered}
$$

где

$$
y_{i j}=\left\{\begin{array}{c}
1, \text { eслих } x_{i j}>0 \\
0, \text { иначе }
\end{array},\right.
$$

другими словами, $y_{i j}=1$, тогда и только тогда, когда происходит отправка продукции из склада $\mathrm{i}$ в пункт $\mathrm{j}$,

$$
z_{j}=\left\{\begin{array}{c}
1, \text { eсли } x_{i j}>0 \\
0, \text { иначе }
\end{array},\right.
$$

другими словами, $z_{j}=1$ тогда и только тогда, когда происходит отправка продукции из склада $i$ в пункт $j$, ограничение (14) утверждает случай, когда происходит отправка продукции по дороге инцидентной складу $i$ и покупателю $j$, тогда $y_{i j}=1$, ограничение (14) служит усилением к ограничению (15). Ограничение (17) не дает задействовать больше чем Q складов.

Подобные задачи часто возникают на любых производственных предприятиях: что производить и в каких объёмах, чтобы суммарная прибыль предприятия была максимальна с учетом минимизации временных и денежных издержек.

Задача $F_{0}$, решена с помощью пакета Matlab. Ответ получим в виде одномерных массивов $X$. Размерность $X=r+2 n^{2}$. Первые $r$ элементов отвечают за количество открытых пунктов складов. Следующие $n^{2}$ переменных - объем перевезенной продукции по каждой дуге. Последние $n^{2}$ элементов отвечают за значения вспомогательных переменных $y$. Рассмотрим ее подробнее.

Существует несколько методов решения таких задач. Среди них можно выделить: Метод Литтла, Метод ветвей и границ, Генетический алгоритм. Рассмотрим их в таблице 2.

Таблица 2

\section{Сравнительные характеристики алгоритмов}

\begin{tabular}{|l|c|c|}
\hline \multicolumn{1}{|c|}{ Признаки сравнения } & Скорость сходимости & Учитывает ли проблему «Big Data» \\
\hline Метод Литтла [5] & Высокая & Нет \\
\hline Метод ветвей и границ [6] & Низкая & Нет \\
\hline Генетический алгоритм [7] & Низкая & Да \\
\hline
\end{tabular}


Метод Литтла представляет собой алгоритм отсечений путем генерации прямых (плоскостей, гиперплоскостей) и введением их в систему ограничений [3]. Метод ветвей и границ представляет собой дерево решений, конечным результатом которой является оптимальное решение [8]. Вышеперечисленные методы являются достаточно быстрыми алгоритмами для задач небольшой выборки. Однако, когда речь идет о проблеме более известной в мире, как «Big Data», становится очевидным тот факт, что эти два метода не позволяют решить задачу. Однако уже в XXI столетии был разработан генетический алгоритм. Ниже приведена общая схема алгоритма [9]. Данный алгоритм особенно хорош, когда мы говорим о задачах линейного программирования (ЛП). Согласно теории [10], допустимое множество решений, а значит, и оптимальное в том числе, есть множество компакт [11] - ограниченное и замкнутое. Как известно, одним из главных минусов этого эвристического алгоритма является тот факт, что существует вероятность нахождения алгоритмом локального минимума и далее застревание в нем. Так как множество допустимых решений является компактом, то очевидно, что за конечное время генетический алгоритм найдет решение линейной задачи, даже если задача будет большой размерности. Сложность этого алгоритма заключается в составлении целевой функции.

Выберем метод Литтла, ввиду быстрой сходимости на небольшой выборке.

Pезультаты и обсужсдение / Results and discussion. Все входные данные представлены в $[12,13]$. На рис. 1 представлена произвольная визуализация графа D. Номера вершин - пункты производства, промежуточные пункты, пункты потребления. Веса дуг матрицы D есть максимальное число единиц продукции, которое можно провезти по каждой дуге.

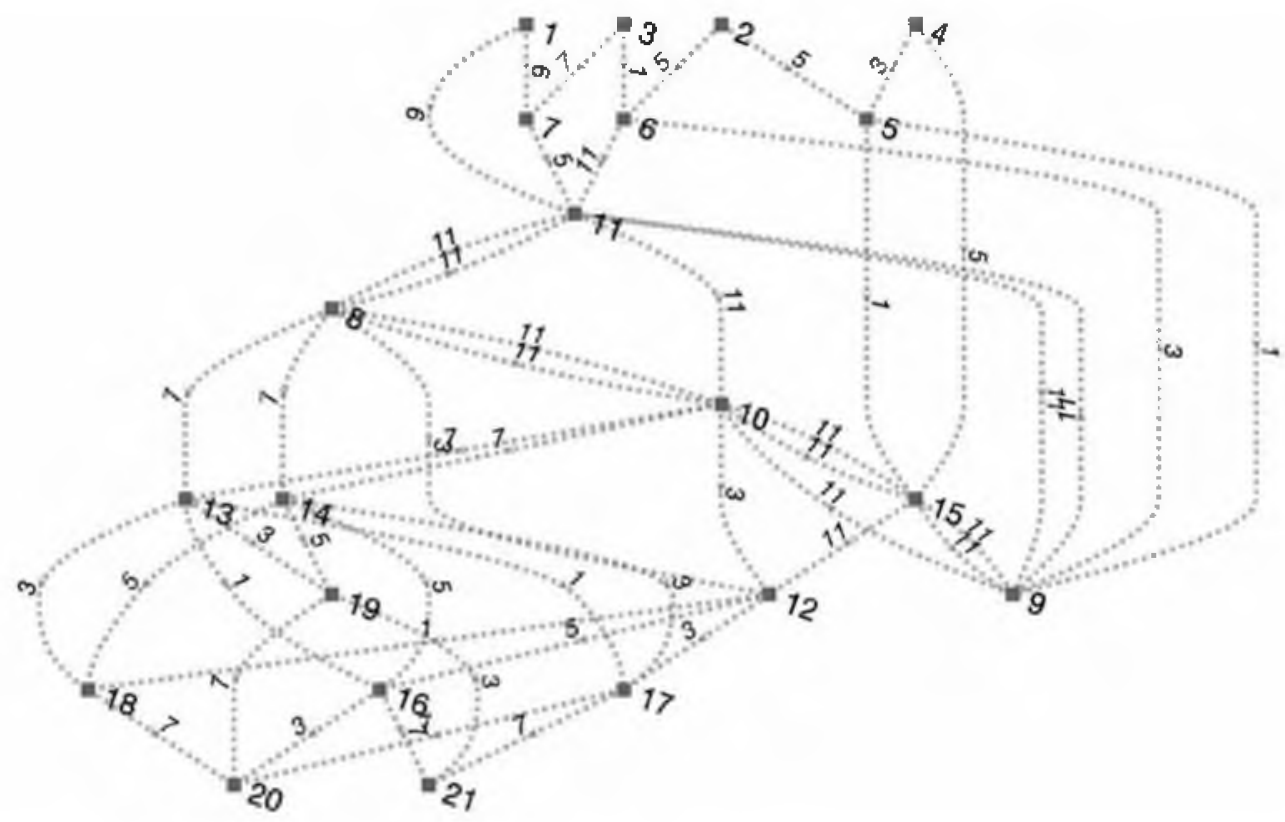

Рис. 1. Произвольная визуализация графа D

Решим задачу с входными данными $[12,13]$.

Таблица 3

Сравнение выходных данных при работе моделей

\begin{tabular}{|l|c|c|}
\hline \multicolumn{1}{|c|}{ Параметр } & Последовательно [12] & Комплексно [13] \\
\hline Объем перевезенной продукции (вектор), шт & 10 & 10 \\
\hline Суммарные затраты на перевозку, у. е. & 3873 & 3469 \\
\hline
\end{tabular}




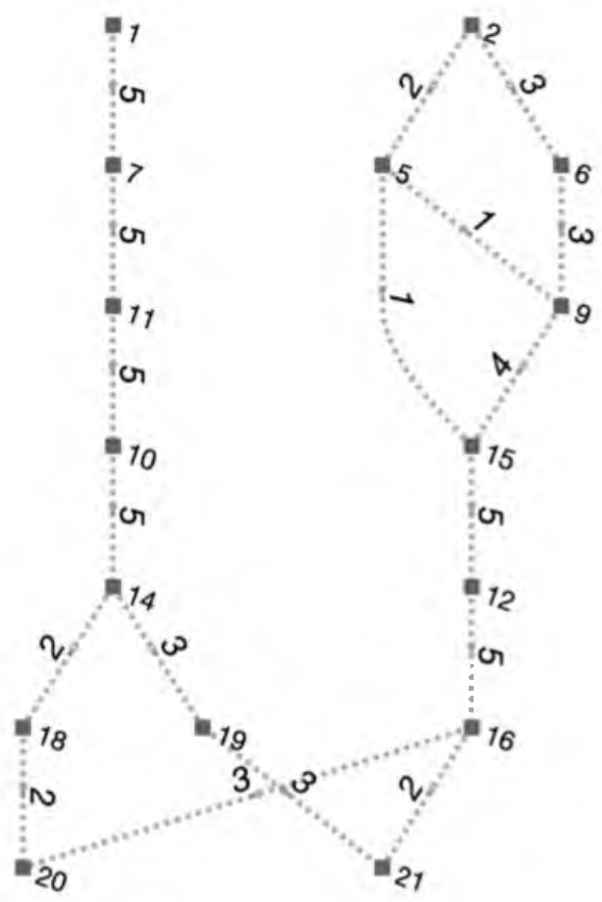

Рис. 2. Визуализация решения рассматриваемой задачи последовательным методом

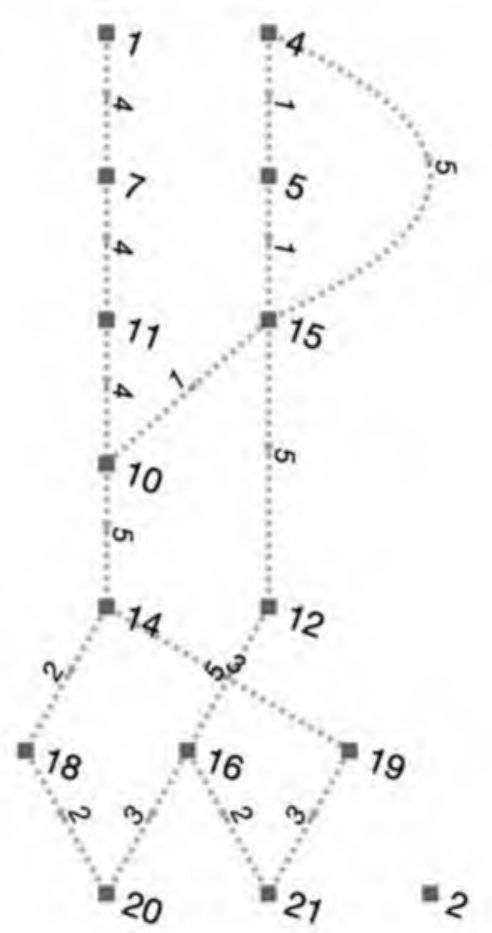

Рис. 3. Визуализация решения рассматриваемой задачи с использованием комплексной модели

Рассмотрим рис. 2 и 3 . На них отчетливо видно, что начинается перевозка из разных пунктов, что влечет за собой разные издержки (таблица 3), во-первых, по открытию нового пункта, во-вторых, по конечному варианту передвижения по графу дорог. Однако стоит заметить, что не 
всегда будут разниться решения, другими словами, будет существовать такой граф, последовательное решение на котором будет совпадать с решением комплексной модели. Этот фактор связан со структурой графа.

Заключение / Conclusion. В данной статье была рассмотрена одна из возможных постановок задачи, которая обобщает ранее известные 3 классические задачи линейного программирования. Было показано, что такую задачу возможно сформулировать в рамках задачи линейного программирования. Решен производственно-логистический пример на 21 вершинах с 4 пунктами входа, 2 пунктами выхода. Показано, что такую задачу возможно решать и визуализировать средствами пакета Matlab. Рассмотрен ряд возможных добавлений ограничений в модель. Такая постановка задачи и модель могут быть использованы на любом предприятии, где необходимо найти оптимальный комбинаторный вариант для производства с целью минимизации логистических издержек в ходе транспортировки.

\section{ЛИТЕРАТУРА И ИНТЕРНЕТ-РЕСУРСЫ}

1. Писарук Н. Н. Исследование операций. Минск: БГУ, 2015. 304 с.

2. Lee J., \& Wiegele A. Another pedagogy for mixed-integer Gomory // EURO Journal on Computational Optimization. 2017. № 5(4). Pp. 455-466.

3. Xiaoping Jiang, Ruibin Bai, Jason Atkin, Graham Kendall. A scheme for determining vehicle routes based on Arc-based service network design // INFOR: Information Systems and Operational Research. 2017. № 55:1. Pp. 16-37.

4. Morrison D. R., Sewell E. C., \& Jacobson S. H. An application of the branch, bound, and remember algorithm to a new simple assembly line balancing dataset. European Journal of Operational Research. 2014. № 236(2). Pp. 403-409.

5. Chu W. S., de la Torre F., Cohn J. F. \& Messinger D. S. A Branch-and-Bound Framework for Unsupervised Common Event Discovery // International Journal of Computer Vision. 2017. № 1-20.

6. Siew Mooi Lim, Abu Bakar Md. Sultan, Md. Nasir Sulaiman, Aida Mustapha, and Leong K. Y. Crossover and Mutation Operators of Genetic Algorithms // International Journal of Machine Learning and Computing. 2017. Vol. 7. No. 1. Pp. 9-12,

7. Sumathi P. () A new approach to solve linear programming problem with intercept values // Journal of Information and Optimization Sciences, 2016. № 37:4. Pp. 495-510.

8. Daganzo C. F. \& Smilowitz K. R. Bounds and approximations for the transportation problem of linear programming and other scalable network problems // Transportation Science. 2004. № 38(3). Pp. 343-356.

9. Hadi Heidari Gharehbolagh, Ashkan Hafezalkotob, Ahmad Makui, and Sedigh Raissi. A cooperative game approach to uncertain decentralized logistic systems subject to network reliability considerations // Kybernetes. 2017. Vol. 46. № 8. Pp. 1452-1468.

10. Maysara Sayed, Linda C. Hendry, Marta Zorzini Bell. Institutional complexity and sustainable supply chain management practices // Supply Chain Management: An International Journal. 2017. Vol. 22. Issue 6. Pp. 542-563. URL: https://doi.org/10.1108/ SCM-10-2016-0365

11. https://pastebin.com/Mz2xnwft

12. https://pastebin.com/8KZ5Bf5j

\section{REFERENCES AND INTERNET RESOURCES}

1. Issledovanie operacij (Operations Research). Minsk: BGU, 2015. $304 \mathrm{~s}$.

2. Lee J., \& Wiegele A. Another pedagogy for mixed-integer Gomory // EURO Journal on Computational Optimization. 2017. № 5(4). Pp. 455-466.

3. Xiaoping Jiang, Ruibin Bai, Jason Atkin, Graham Kendall. A scheme for determining vehicle routes based on Arc-based service network design // INFOR: Information Systems and Operational Research. 2017. № 55:1. Pp. 16-37.

4. Morrison D. R., Sewell E. C., \& Jacobson S. H. An application of the branch, bound, and remember algorithm to a new simple assembly line balancing dataset. European Journal of Operational Research. 2014. № 236(2). Pp. 403-409. 
5. Chu W. S., de la Torre F., Cohn J. F. \& Messinger D. S. A Branch-and-Bound Framework for Unsupervised Common Event Discovery // International Journal of Computer Vision. 2017. № 1-20.

6. Siew Mooi Lim, Abu Bakar Md. Sultan, Md. Nasir Sulaiman, Aida Mustapha, and Leong K. Y. Crossover and Mutation Operators of Genetic Algorithms // International Journal of Machine Learning and Computing. 2017. Vol. 7. No. 1. Pp. 9-12,

7. Sumathi P. () A new approach to solve linear programming problem with intercept values // Journal of Information and Optimization Sciences, 2016. № 37:4. Pp. 495-510.

8. Daganzo C. F. \& Smilowitz K. R. Bounds and approximations for the transportation problem of linear programming and other scalable network problems // Transportation Science. 2004. № 38(3). Pp. 343-356.

9. Hadi Heidari Gharehbolagh, Ashkan Hafezalkotob, Ahmad Makui, and Sedigh Raissi. A cooperative game approach to uncertain decentralized logistic systems subject to network reliability considerations // Kybernetes. 2017. Vol. 46. № 8. Pp. 1452-1468.

10. Maysara Sayed, Linda C. Hendry, Marta Zorzini Bell. Institutional complexity and sustainable supply chain management practices // Supply Chain Management: An International Journal. 2017. Vol. 22. Issue 6. Pp. 542-563. URL: https://doi.org/10.1108/ SCM-10-2016-0365

11. https://pastebin.com/Mz2xnwft

12. https://pastebin.com/8KZ5Bf5j

\section{СВЕДЕНИЯ ОБ АВТОРАХ}

Рогулин Родион Сергеевич, магистрант 2-ого курса направления «Прикладная математика и информатика», Дальневосточный федеральный университет, г. Владивосток. E-mail: rafassiaofusa@mail.ru

Максименко Валерий Иванович, кандидат технических наук, доцент, доцент, доцент кафедры гидротехники, теории зданий и сооружений, Дальневосточный федеральный университет, г. Владивосток. E-mail: maximenko.vi@dvfu.ru

Жапдариов Владимир Олегович, магистрант 1-ого курса направления «Кибербеезопасность», Дальневосточный федеральный университет, г. Владивосток. E-mail: zhandarmov.vo@students.dvfu.ru

Пугачева Ева Сергеевна, студентка 2-го курса, направления «Медицинская биофизика, кибернетические и биотехнические системы», Дальневосточный федеральный университет, г. Владивосток. E-mail: pugacheva.es@students.dvfu.ru

Матвеев Владислав Викторович, студент 3-курса, направления «Биотехнология», Дальневосточный федеральный университет, г. Владивосток. E-mail: matveev.vv@students.dvfu.ru

Рогулин Данила Серzеевич, студент бакалавр 1-го курса, направления «Прикладная информатика», Владивостокский государственный университет экономики и Сервиса, г. Владивосток. E-mail: rogulin $a$ list.ru

\section{INFORMATION ABOUT AUTHORS}

Rodion Rogulin, 2nd year undergraduate student in the field of Applied Mathematics and Computer Science, Far Eastern Federal University, Vladivostok. E-mail: rafassiaofusa $@$ mail.ru

Valery Maksimenko, Ph.D., Associate Professor, Associate Professor of the Department of Hydraulic Engineering, Theory of Buildings and Structures, Engineering School, Far Eastern Federal University, Vladivostok. E-mail: maximenko.vi@dvfu.ru

Vladimir Zhandarmov, First-year Undergraduate in Cyber Security, Far Eastern Federal University, Vladivostok. E-mail:zhandarmov.vo@students.dvfu.ru

Eva Pugacheva, 2nd year student, directions «Medical Biophysics, Cybernetic and Biotechnical Systems», Far Eastern Federal University, Vladivostok. E-mail: pugacheva.es@students.dvfu.ru

Vladislav Matveev, 3rd year student, Biotechnology, Far Eastern Federal University, Vladivostok. E-mail: matveev. vv@students.dvfu.ru

Danila Rogulin, 1st year bachelor's student, Applied Informatics, Vladivostok State University of Economics and Service, Vladivostok. E-mail: rogulinalist.ru 